\title{
De la crise économique à la critique de la science économique
}

Une présentation de l'ouvrage d'André Orléan, L'Empire de la valeur (Seuil, 2011)

Ivan Jaffrin

\section{OpenEdition}

\section{Journals}

Édition électronique

URL : http://journals.openedition.org/ress/2305

DOI : $10.4000 /$ ress.2305

ISSN : 1663-4446

Éditeur

Librairie Droz

\section{Édition imprimée}

Date de publication : 1 décembre 2012

Pagination : 197-207

ISBN : 978-2-600-01704-6

ISSN : 0048-8046

\section{Référence électronique}

Ivan Jaffrin, « De la crise économique à la critique de la science économique », Revue européenne des sciences sociales [En ligne], 50-2 | 2012, mis en ligne le 15 décembre 2012, consulté le 22 septembre 2020. URL : http://journals.openedition.org/ress/2305 ; DOI : https://doi.org/10.4000/ress.2305 


\title{
AUTOUR DE L'OUVRAGE D'ANDRÉ ORLÉAN L'EMPIRE DE LA VALEUR - REFONDER L'ÉCONOMIE
}

Paris, Seuil, «La couleur des idées», 20II

\author{
DE LA CRISE ÉCONOMIQUE \\ À LA CRITIQUE DE LA SCIENCE ÉCONOMIQUE \\ Une présentation de l'ouvrage d'André Orléan, L'Empire de la valeur (Seuil, 20II)* \\ par Ivan Jaffrin \\ Revue européenne des sciences sociales, Librairie Droz
}

C'est l'une des singularités de l'«économie» - et pas la moindre de ses complications - que de désigner tout à la fois la science et son objet: l'économie n'est rien d'autre en effet que la science de... l'économie. La définition classique de la discipline économique, entendue comme science de l'allocation optimale des ressources rares, ne change d'ailleurs rien au fait qu'il ne saurait y avoir dans la réalité, non plus que dans la nature, de référent répondant spontanément au nom d'«allocation des ressources rares». Un tel objet n'est jamais qu’un état du monde social appréhendé sous le rapport économique, et donc à l'aune de la science économique elle-même. On pourrait certes en dire autant de l'ensemble des disciplines des sciences sociales, dont le regard que chacune d'elles porte sur son objet participe de leur construction respective. Cependant, dans le cas précis de l'«économie», il y a, pour ainsi dire, superposition de l'ordre des faits économiques au fait de l'ordre économique, au point qu'il devient hasardeux de discerner entre les deux et que la faillite du premier sonne comme le démenti nécessaire du second.

Tel est semble-t-il le point de départ de l'ouvrage d'André Orléan, L’Empire de la valeur, qui entend précisément partir du constat du discrédit dont pâtit la discipline économique à la suite de la crise financière de 2007-2008 pour justifier son entreprise de refondation théorique, dont l'ambition est d'emblée déclarée en sous-titre de l'ouvrage. L'auteur prend en effet acte avec vigueur de ce que la financiarisation de l'économie - dont on s'accorde à dire qu'elle est en grande partie responsable de la crise - est elle-même un produit de la science économique (EV, p. 322). C’est que «la théorie économique joue un rôle immense dans nos sociétés développées. Elle est le discours qui indique comment les affaires humaines doivent être menées» (EV, p. III). Or c'est là, selon l'auteur, membre du collectif des «économistes atterrés» et fondateur de l'Association française d'économie politique, une anomalie de la science économique qui la distingue du reste des sciences sociales. «Refonder l'économie», cela doit donc consister, pour commencer, par en «finir avec le séparatisme qui caractérise l'économie en tant

$*$

EV dans toute la suite du dossier. 
que discipline » (EV, p. I89). Pour ce faire, l'auteur entend bien s'efforcer à surmonter le schisme entre le raisonnement économique et le raisonnement sociologique en défendant l'idée que «le fait économique est un fait social comme un autre » (EV, p. I86). De ce point de vue, c'est moins le «capitalisme» qui est au cœur de l'étude d’André Orléan que l'économie marchande au sens large, à travers les pratiques d'échange et les modalités de définition de la valeur auxquelles elle donne lieu.

D’inspiration hétérodoxe - l'auteur est partisan des théories de la régulation et d'une «économie des conventions» assez minoritaire dans le panorama des sciences économiques -, André Orléan se livre à une critique de la notion de valeur dans les théories classiques et néoclassiques dont la portée est considérable. Il s’agit rien moins que de faire valoir dans l’analyse des pans entiers de la réalité indûment laissés de côté par la modélisation économique. Couronné, à la fin de l'année 20ı i, par le prix Paul Ricœur, L'Empire de la valeur est assurément un ouvrage important qui constitue l'une des premières tentatives rigoureuses et systématiques de repenser l'économie après la crise. Que ce prix soit celui d'un philosophe n'a en l'espèce rien d'étonnant tant l'ouvrage donne lieu à une réflexion théorique très ambitieuse. Visant à élaborer une théorie de la valeur en économie qui soit de nature à préserver l'unité des sciences sociales, il constitue une très remarquable illustration des vertus de l'interdisciplinarité.

André Orléan développe une réflexion en trois temps qui progresse suivant une subdivision en sept chapitres que nous nous proposons de suivre pas à pas : dans une première partie (Critique de l'économie), il s'emploie à mettre à jour le caractère mystificateur d'une valeur conçue comme substance, tant dans les théories classiques que néoclassiques et marginalistes. Ce faisant, il se livre à une vigoureuse contestation de l'«objectivité marchande » qui pose quau commencement était la rareté. Dans une deuxième partie, il entend établir que c'est le fait monétaire qui est premier dans l'ordre des phénomènes économiques et pose le primat des rapports sociaux dans la détermination de la valeur (L'institution de la valeur). Enfin, dans une troisième partie, l'auteur se penche sur le cas d'espèce de la finance pour souligner tout le caractère erroné de la théorie de l'efficience des marchés financiers (La finance de marché).

\section{D’une économie des grandeurs à une économie des relations}

Le raisonnement économique a pour fondement l'idée que la relation marchande élémentaire repose sur le principe de l'équivalence en valeur (EV, p. 20). Deux biens quelconques sont ainsi échangés sur un marché en vertu d'une valeur objective qu'ils auraient en propre et qu'il appartient à l'échange marchand de déterminer à travers la fixation d'un juste prix. Ce que l'auteur appelle le monde de la «séparation marchande», c'est alors celui d'un ordre où des producteurs-échangistes, isolés les uns des autres, se coordonnent entre eux par la seule médiation de la marchandise. Il en résulte que «la sphère économique accède à une existence séparée, indépendante des autres activités sociales » (EV, p. I2) où, à 
l'exemple des sciences de la nature, seule compte la mesure des choses. Mais d'où vient une telle objectivité de la valeur, s'interroge André Orléan [Chap. I. La valeur substance: travail et utilité]? Tandis que les pères fondateurs de la science économique (Smith, Ricardo et Marx) l'ont placée dans le facteur de production en indexant la valeur d'un bien sur la quantité de «travail» requise pour le produire, les néoclassiques (Jevons, Menger, Walras) l'ont faite porter dans sa propension à satisfaire une «utilité » pour le consommateur.

Il est à cet égard remarquable que les uns comme les autres, par delà leur divergence de vue, ont finalement en commun de faire fond sur une théorie implicite de la valeur, sans équivalent dans les autres sciences sociales, qui pose que cette dernière réside dans les choses elles-mêmes. L'auteur montre alors combien cette «hypothèse substantielle» (EV, p. 24) est redevable d'une vision de l'économie qui tend à naturaliser le phénomène économique en faisant du troc l'archétype de l'échange marchand. Pour autant que la monnaie n'y est pas absente, elle est en effet pour le moins transparente : elle tient lieu de médiation superfétatoire visant simplement à faciliter les transactions sans altérer aucunement la nature de l'échange (EV, p. 25). Or, une telle vision procède, selon l'auteur, d'une croyance en l'objectivité de la valeur par la médiation du prix qui sous-estime grandement l'importance des rapports sociaux dans l'échange marchand et qui, de surcroît, n'est en aucun cas conforme aux données de l'expérience.

Ceci est tout particulièrement vrai du modèle de l'équilibre général walrassien qu'André Orléan entreprend d'examiner avec grand soin, tant il demeure aujourd'hui encore la forme canonique de la relation marchande dans la science économique [Chap. 2. L'objectivité marchande]. Le prix d'équilibre y est conçu comme l'expression de la valeur intrinsèque de la marchandise. C'est par le biais de cette «médiation externe » parfaite (EV, p. 90-9I) que l'offre et la demande s'égalisent jusqu'au point où tous les marchés sont à l'équilibre simultanément et où la satisfaction de chaque agent est portée à son maximum. Seule importe, dans ce cadre, la relation de l'individu aux biens qui sont échangés, et nullement sa relation aux autres. C'est ainsi qu'en situation de concurrence pure et parfaite, souligne Walras, il ne saurait y avoir de relations entre les participants au marché (EV, p. 66-67) : toute l'information disponible transite par l'intermédiaire du «secrétaire de marché » (ou «commisseur-priseur ») qui seul détermine les prix d'équilibre. À la limite, ironise André Orléan, il ne reste plus aux acteurs, dans le modèle walrassien, qu'à effectuer les transactions; lesquelles tendront sans surprise, à la faveur du libre jeu de la concurrence, vers l'équilibre général, puisque la totalité de l’incertitude aura été préalablement résorbée dans le prix.

Il ne faut pas s'y tromper, le modèle walrassien n'est jamais qu'une «vue de l'esprit» certes puissante mais qui ne saurait prétendre à une pertinence étendue à toute la réalité. En tant qu'idéal-type, concède l'auteur, il vise moins une approximation du monde réel qu'une stylisation de certaines de ses tendances, et par conséquent ne décrit pas tant des «comportements moyens» que des «comportements typiques» (EV, p. Io9-I Io). Et 
André Orléan de rappeler tous les cas de figure qui invalident la thèse de l'utilité marginale décroissante au fondement de la théorie de l'équilibre général («quête du prestige» telle que décrit par Veblen, [EV, p. I30]; «théorie des rendements croissants » d’Arrow et Debreu, [EV, p. 84]) ou retourne la «loi» de l'offre et de la demande en faisant de cette dernière une fonction croissante du prix («théorie des asymétries d'information» de George Akerlof, Michael Spence et Joseph Stiglitz, [EV, p. 87]).

En pareilles situations, le marché n'est plus autorégulateur et l'équilibre obtenu ne correspond donc pas nécessairement à l'allocation optimale des ressources. Mais, pour autant que la théorie de l'équilibre général a progressivement été amendée - et l'objectivité des préférences des acteurs restreinte à certaines conditions particulières du marché -, la science économique n’en est pas moins restée tributaire d'une perception de l'objectivité marchande qui tient que la valeur économique est indexée sur une utilité consubstantielle aux biens. Quoiqu'elle tolère certaines variations, la mesure de l'utilité ainsi comprise demeure en dernier ressort attachée au phénomène primordial de la rareté et n'est donc nullement conçue comme une fonction des rapports sociaux ou des relations interpersonnelles [Chap. 3. La Rareté].

Or, c'est précisément contre la persistance d'une telle vision de l'économie qu'André Orléan entend opérer un renversement théorique de grande ampleur qui consomme l'abandon d'une «économie des grandeurs» - où la valeur-substance préexiste à l'échange - à la faveur d'une «économie des relations » où ce qui est premier, dans l'ordre des phénomènes, ce sont les rapports sociaux (EV, p. I I5). Ce faisant, il s'agit moins de contester toute pertinence au modèle walrassien que de le subordonner à une théorie globale de la valeur non substantialiste. Et cela revient in fine à limiter son périmètre de validité au seul rapport d'utilité qui n'est jamais qu'une modalité particulière de l'échange et nullement, loin s'en faut, le rapport le plus vraisemblable.

\section{Le désir de monnaie comme fondement de l'échange marchand}

C'est à ce point de l'analyse que le propos de l'auteur revêt son caractère le plus profondément original. André Orléan sollicite alors la philosophie de René Girard pour faire valoir une anthropologie alternative à celle qui a cours dans la science économique - laquelle postule un Homo oeconomicus, isolé et calculateur qui sait ce qu'il désire (conformément à l'hypothèse de l'«exogénéité des préférences », [EV, p. I23]). L'homme-en-relation dont il fait le portrait est, a contrario, un individu dont les préférences sont affectées par celles des autres, lesquelles font, de ce fait, l'objet de rétroactions positives. À l'appui de la théorie de la rivalité mimétique, l'auteur montre ainsi que les individus ne savent pas ce qu'ils désirent, et que loin de préexister à l'échange marchand, les préférences individuelles en sont en fait le produit. 
Ce qui importe dans la relation mimétique, c'est que l'échange marchand n’est pas tant une réalité sui generis que le lieu même où se constitue la valeur dans la relation à l'autre (EV, p. II5 et 139). Il faut donc comprendre que la rivalité n’est pas le fruit de la rareté, comme le veut une tradition bien établie, mais que c'est l'inverse qui est vrai (EV, p. I36) : c'est le phénomène de la rivalité qui génère la rareté en concentrant le désir mimétique sur un nombre limité de biens. À cet égard, l'utilité elle-même n'est jamais qu'une création de l'ordre marchand qui vise à le stabiliser et non une donnée préalable à l'échange (EV, p. II9 et 263). La médiation à la valeur n'est plus dès lors conçue comme externe (incarnée dans les choses, exprimée par le prix), mais endogénéisée: i.e. pensée comme interne (fonction du rapport aux autres, exprimée par le désir).

L’oblitération du fait monétaire par la théorie néo-classique prend alors une signification bien particulière en ce qu'elle exprime le déni du caractère global de la valeur. Parce qu'elle est cette marchandise particulière qui a la propriété unique de pouvoir en donner l'accès à toutes, la monnaie est en effet ce «désir-maître » qui exerce un attrait absolu sur les acteurs. Aussi, loin d'être un expédient visant à faciliter les échanges, la monnaie s'impose-t-elle, selon André Orléan, comme «l'institution première des économies marchandes» (EV, p. I48) [Chap. 4. La monnaie]: elle est «ce par quoi la valeur marchande accède à l'existence» (EV, p. I3). Une économie marchande est ainsi avant toute chose, selon l'auteur, une «économie dans laquelle les acteurs sont à la recherche de monnaie» (EV, p. I49-150). La prise en compte d'un tel affect positif pour l'argent n'aurait rien en soi de très original - André Orléan rappelle qu'elle est déjà contenue dans la mauvaise chrématistique d’Aristote (EV, p. I5 I) -, si l'auteur s'en tenait à la réprouver en condamnant moralement l'«amour de l'argent» et les conduites de type pléonexique qu'il génère. Or il n’en est rien, et l'auteur montre qu'il ne s'agit pas tant là d'une perversion de l'échange marchand, comme le soutient toute la morale utilitariste, que d'une forme d'élan vital qui en est à l'origine. Certes, l'individu mimétique est sujet à l'envie, à la jalousie, à la cupidité, etc. - autant de vices qui font défaut à l'individu rationnel néoclassique - cependant son désir de monnaie n’est pas aberrant puisqu'il est, selon André Orléan, l'expression d'une inclination plus fondamentale inscrite dans la nature même de l'échange: le désir de liquidité.

En tant qu'elle est la puissance marchande par excellence, la liquidité ne renvoie à rien d’autre qu'à elle-même et répond par conséquent à une logique autoréférentielle qui porte la rivalité mimétique à son plus haut degré d'intensité. Est, en effet, liquide «ce que les autres considèrent comme liquide» (EV, p. I55), en sorte que la liquidité n'est pas tant un attribut de la monnaie qu'une fonction essentielle de la confrontation marchande. Et à la limite, n'importe quel bien peut s'en trouver pourvue dès lors que les acteurs lui confèrent un certain «pouvoir d'achat». Cependant, ce que donne à voir la dynamique mimétique, c'est précisément la convergence du désir des acteurs sur un objet unique: la monnaie (EV, p. I56-I58). C'est elle qui capte le désir unanime d'accéder à la puissance 
marchande. L'élection d'une monnaie est ainsi, soutient André Orléan, le résultat nécessaire et inéluctable de la polarisation mimétique qui constitue ce moment fondateur où les acteurs accèdent à une définition unanime du valoir (EV, p. 168-170). Une référence ayant été construite, les préférences des acteurs cessent alors de fluctuer et de stratégique qu'il était en situation d'autoréférence, le phénomène mimétique redevient finalement purement tactique et routinier (EV, p. 86). En d'autres termes, une fois la valeur investie dans l'objet monétaire, elle «se trouve reconnue et désirée par tous» (EV, p. 190) et l'échange marchand peut enfin accéder à une certaine stabilité.

On remarquera au passage qu'il est pour le moins paradoxal de faire de l'élection monétaire l'artefact des échanges marchands tout en postulant une antériorité au fait monétaire qui verrait la rivalité mimétique entre les acteurs pour l'accaparement de la puissance marchande. N'est-ce pas là également un moment constitutf du rapport marchand, quoique non médiatisé par la monnaie? André Orléan le reconnaît (EV, p. 156) qui se défend pourtant d'avoir réintroduit implicitement une situation de troc quand il en dénonçait précisément la prégnance dans la pensée néoclassique. Cette antériorité d'une situation «sans monnaie» est pour lui purement hypothétique, puisqu'il s'agit de montrer au contraire qu'elle renvoie à une situation par nature instable et qui ne saurait durer, où coexistent des représentations concurrentes de la liquidité (EV, p. 159). Et, en effet, qu'on prive de sa monnaie une économie développée et on la verra réapparaître spontanément, comme le produit nécessaire de la polarisation mimétique (EV, p. 160). Mais quid, alors, du troc en tant que modalité historique particulière de l'échange marchand, si c'est le désir de monnaie qui est premier? Il semble bien que ce soit l'un des angles morts de la théorie de l'institution de la valeur que de faire de l'hypothèse du troc une forme implicite de contre-factualité, là où la pensée néoclassique l'avait pensé comme une factualité première, à l'origine des échanges marchands. On se contentera à cet égard d'observer qu'une telle oblitération du troc est sans doute la conséquence inévitable d'une anthropologie qui fait la part belle au phénomène de la rivalité, à l'exclusion de tout autre régime d'action et de coordination.

En définitive, du point de vue d'une économie des relations, le prix d'un bien, loin d'être l'expression d'une valeur qui lui préexisterait, égale la valeur que lui reconnaissent les autres à l'issue de l'interaction mimétique. André Orléan parachève alors son raisonnement à la faveur d'une théorie du fait monétaire particulièrement audacieuse qui a pour effet, conformément à son ambition initiale, de ramener la science économique dans le giron des sciences sociales - et plus précisément dans celui de la sociologie de Durkheim, à travers son analyse du fait religieux [Chap. 5. Un cadre unidisiplinaire pour penser la valeur]. Car ce que réalise la monnaie dans l'échange, c'est au fond la «capture de l'affect commun» au sens où Durkheim avait définit le totémisme (EV, p. 209). La liquidité est à cet égard décrite comme un analogon de cette force impersonnelle qu'est le «mana» chez les Mélanésiens, dont il est question dans Les Formes élémentaires de la vie religieuse. La liquidité 
est pour ainsi dire «surajoutée» à la monnaie, comme le sacré l'est au fétiche, qui se trouve pourvu d'emblée d'une autorité unanimement reconnue comme telle.

André Orléan se défend cependant de vouloir amalgamer la valeur économique à la valeur religieuse. Il entend au contraire rendre compte d'une autonomisation des valeurs économiques qui ont constitué un ordre antinomique à l'ordre moral et religieux (EV, p. 220). À travers le fait monétaire, ce qu'il convient de souligner c'est finalement la toute puissance de l'auto-extériorisation du social qui est, de manière analogue au fait religieux, générateur de croyances collectives autonomes. Initialement conçue comme interne au rapport mimétique, la valeur est pour finir médiatisée par une convention externe aux acteurs qui n'est nullement fonction d'une quelconque substance déjà-là. On comprend dès lors, l'inquiétude qui animera les penseurs libéraux dès qu'il s'agira de penser la monnaie. C'est qu'elle est fondamentalement, nous dit André Orléan, cette autorité première - scandaleuse du point de vue de l'individualisme méthodologique -, qui donne à voir la «société en tant que totalité » (EV, p. 2I7) et tient en conséquence le langage de la souveraineté (EV, p. 2I4). D'où l'obsession de quelques-uns des libéraux les plus conséquents d'en neutraliser autant que faire se peut les effets potentiellement déstabilisateurs (ainsi de Rueff et Friedman, partisans de l'indépendance des banques centrales ou de Hayek, [EV, p. 217]). D’où aussi l'expression récurrente de «miracle économique » pour caractériser certains phénomènes monétaires bien connus (EV, p. 22I), explication pour le moins incongrue dans le champ des sciences sociales mais qui n'est peut-être pas étrangère à ces fameux «chocs de confiance » dont il est souvent question aujourd'hui. Car, en définitive, l'institution de la valeur est essentiellement affaire de confiance : ce qui fonde la valeur c'est le crédit qu'on porte à son expression monétaire, laquelle n'est jamais qu'une convention, une croyance collective. Qu'on cesse de vouloir s'échanger une monnaie, et la voilà qui cesse de facto d'être liquide (EV, p. IO2). Une crise économique est de ce point de vue une défiance des conventions passées, un discrédit momentané de la valeur (EV, p. I8I-I84).

\section{L'emprise de la liquidité sur les marchés financiers}

Parce que la liquidité induit une logique autoréférentielle où les préférences des acteurs ne sont pas fixées mais dépendent les unes des autres, elle est source d’une grande instabilité. En ce sens, le phénomène de la liquidité transforme profondément la logique walrassienne. Or, ceci vaut de manière exemplaire, selon André Orléan, pour le marché financier auquel il consacre l'ultime partie de son ouvrage. Il s'agit là du volet le plus spécifique de sa démonstration, dont on imagine aisément qu'il est à l'origine même de son projet de refondation théorique. En soumettant l'évaluation financière à l'épreuve de l'hypothèse mimétique, l'auteur entend en effet battre en brèche la thèse de l'efficience des marchés financiers [Chap. 6. L'évaluation financière]. Pas plus qu'il n'y a de valeur-substance des biens, il n’y a de valeurs fondamentales des titres, indépendantes 
des transactions financières. Le caractère erratique des cours en certaines circonstances et les aberrations manifestes auxquelles ont pu donner lieu, à de multiples reprises, les évaluations financières, devraient suffire à s'en convaincre. La crise de 2007-2008, tout comme auparavant celle de la bulle Internet, a ainsi montré avec éclat le caractère pour le moins contestable de l'image d'un marché financier autorégulateur.

L'erreur fondamentale à ne pas commettre dans l'analyse des marchés financiers consiste ainsi à croire, selon André Orléan, qu'il y aurait un futur objectivable (qu'il appelle «hypothèse de nomenclature des états du monde », [EV, p. 250]), tel que les anticipations rationnelles des acteurs pourraient être forgées en fonction d'un risque mesurable et connu de tous. Récusant une telle approche probabiliste, l'auteur montre au contraire, à l'appui des thèses de Frank H. Knight, qu'il est des situations d'incertitudes radicales où l'on ne peut faire l'impasse sur le caractère irréductible de la subjectivité des estimations individuelles. Ceci est particulièrement vrai lorsque surviennent des événements uniques dont il n'est pas possible de calculer la fréquence et au devant desquels l'acteur est livré à sa seule faculté de juger (EV, p. 254). Or, loin d'être exceptionnelle, la survenance de tels événements constitue au contraire l'occurrence la plus vraisemblable ainsi qu'en témoigne, de manière exemplaire, l'émergence de la nouvelle économie de l'Internet qui donna lieu aux cotations les plus contradictoires.

Aussi, ce que feint d'ignorer la théorie financière, c'est finalement l'emprise de la liquidité sur le monde financier par la cotation permanente des prix, telle que les «évolutions de prix, même à court terme, exercent une véritable tyrannie sur les comportements financiers» (EV, p. 270) [Chap. 7. Liquidité et spéculation]. En effet, en tant qu'un actif financier est liquide, il fait l'objet de transactions sur le marché boursier et connaît des «variations constantes de prix, qui sont autant d'occasions de profits pour les échangistes » (EV, p. 268). Mais il s'agit-là de profits issus des transactions elles-mêmes et non pas de projections sur le long terme, attachées à une quelconque valeur fondamentale du titre. D'où la tendance naturellement haussière du marché financier porté à générer des bulles spéculatives. Nulle irrationalité des marchés cependant car, au fond, «la rationalité spéculative est la rationalité qu'exige la liquidité» (EV, p. 277).

En l'absence de tout ancrage extérieur, l'évaluation financière donne ainsi à voir un phénomène par nature autoréférentiel et mimétique : parce que les prix qui se forment résultent d'anticipations collectives de prix futurs, il revient à tout un chacun de «mimer le marché afin de le précéder dans son évolution» (EV, p. 27I). Il faut donc comprendre que, pas plus que la valeur ne préexiste à l'échange, le «futur» ne préexiste aux actions individuelles. Il en est également le produit: «les évaluations des investisseurs sanalysent comme des paris concernant un futur, qui non seulement n'est pas écrit, mais qui surtout dépend étroitement des paris qui auront été faits sur lui» (EV, p. 25I). Et André Orléan de pointer alors le caractère profondément spéculaire de la spéculation : c'est 
qu'il s'agit fondamentalement pour les opérateurs financiers de se forger «une croyance qui a en fait pour objet la croyance des autres participants» (EV, p. 282).

Ce jeu de «pure coordination» est examiné en détail et de manière très convaincante par l'auteur à partir de la thèse de l'autonomie des saillances de Thomas Schelling (EV, p. 284-289). Il en retient une idée tout à fait essentielle, à savoir que la croyance collective qui se forme à l'issue de la polarisation mimétique n'est en réalité la croyance de personne (EV, p. 290). Elle tient leu de convention par défaut visant à permettre la coordination d'un collectif en l'absence d'une référence commune préalablement constituée. Aussi, loin de freiner la dérive des cours, le phénomène mimétique est-il au contraire de nature à l'amplifier à la faveur de tendances conventionnelles à la hausse comme à la baisse sans commune mesure avec la réalité économique. L’autoréférentialité née de la liquidité des titres conduit en conséquence à des variations extrêmes des prix rendant pour ainsi dire justice à l’adage qui veut que «passée la mesure, il n’y a plus de limites »...

\section{Conclusion}

L’ouvrage d'André Orléan a l'insigne mérite de s’attacher à produire une théorie économique qui soit, à la différence du modèle walrassien et néoclassique, d'abord attentive à l'instabilité des échanges marchands et financiers. Inspiré par le souci d'infléchir la science économique dans le sens d'une plus grande conformité aux faits, l’auteur en vient enfin à tirer quelques conclusions programmatiques. Ayant ainsi établi que plus le marché financier est liquide, plus les comportements des acteurs relèvent d'une stratégie mimétique fortement déstabilisatrice, il en déduit qu'il convient de rendre le marché moins liquide et qu’en conséquence la «dérégulation est une faute majeure » (EV, p. 297). C'est la conséquence logique d’une condamnation de la spéculation «déconnectée de l'économie réelle » qui n’a certes rien de très originale - et qui ne surprendra pas de la part d'un économiste d'inspiration régulationniste -, mais qui n’est pas sans reposer de manière aiguë la question d'une taxe sur les flux financiers. De même, on ne s'étonnera guère de voir André Orléan, partisan de la théorie des conventions et d'une monnaie conçue comme «souveraine dans l'ordre marchand» (EV, p. 2I2), critiquer l'indépendance de la Banque centrale européenne, coupable à ses yeux de tendre vers l'objectif illusoire de la neutralisation monétaire (EV, p. 215). Pour n'être pas renversantes en termes d'originalité, ces conclusions n'en sont pas moins remarquables en ce qu'elles ne relèvent pas de mesures incantatoires mais procèdent d'une démonstration qui leur confère la force de la nécessité. Et ce n'est d’ailleurs pas la moindre des qualités de cet ouvrage que d'exposer avec une grande clarté une pensée d'une rare tenue, à la faveur d'une théorie de la valeur pour le moins ambitieuse qui renouvelle avantageusement les analyses de Marx et de Keynes, auxquelles l'auteur doit manifestement beaucoup. 
Mais si la grande cohérence du propos est une force qui convainc intellectuellement, saura-t-elle pour autant séduire les salles de marchés ou influencer la manière d'enseigner la pratique de l'économie? Il est hélas permis d'en douter, et c'est aussi l'un des mérites supplémentaires de cet ouvrage que d'envisager les raisons d'une telle imperméabilité de l'économie aux acquis des sciences sociales et aux démentis de la réalité. André Orléan fait référence, en conclusion à son ouvrage, à la fameuse querelle qui opposa Léonard Savage à Maurice Allais sur le point de savoir comment se comportent les acteurs en situation d'incertitude. Ce qui est désormais connu sous le nom de «paradoxe d'Allais » constitue à cet égard une remise en cause patente de la loi de «maximisation de l'espérance d'utilité » et de transitivité des préférences que soutient Savage, lequel tombe lui-même dans le piège du paradoxe puisqu'il est amené à devoir soutenir une préférence en contradiction avec sa propre théorie. Savage ne se démonte pas pour autant, qui, tout en reconnaissant de bonne foi son erreur, préfère en définitive changer de choix plutôt que de théorie... Et André Orléan de rappeler alors opportunément que François Simiand s'insurgeait déjà contre une telle pratique consistant à imputer l'écart entre le modèle et la réalité non pas à une erreur du modèle mais à une défaillance de la réalité (EV, p. 317 ). C’est que l'individu rationnel postulé par la pensée néoclassique ne saurait exprimer de préférences autres que celles que lui dicte le modèle de la rationalité utilitaire, en sorte que s'il devait vouloir autre chose que la maximisation de son utilité, il reviendrait à la science économique le rôle impérieux de le détromper. Là réside sans doute l'un des vices les plus tenaces de la théorie économique qui n’hésite pas à influer directement sur le comportement des individus pour peu qu'il en aille de la conformité des faits aux prérequis de la théorie. C'est comme si la pierre qui tombe se référait directement à la loi de la gravité pour déterminer sa trajectoire, fait mine de s'étonner André Orléan: «ce n’est plus la gravité qui la meut mais son adhésion au modèle newtonien» (EV, p. 320). Ainsi des agents économiques qui veillent à ne pas déroger dans leur comportement aux attendus de la science économique et qui, ce faisant, donnent l'illusion de la vérifier à chaque instant. Une telle «performativité» de la science économique, qui génère des comportements à son image, justifie en définitive l'entreprise menée par l'auteur visant à modifier cette dernière aux fins d'induire un changement dans les comportements financiers dans un sens sinon plus rationnel, du moins plus raisonnable. Toute la question reste de savoir s'il est vraiment dans l'intérêt des marchés boursiers que de s'amender de la sorte, et si l'écho des thèses refondatrices d'André Orléan sera en mesure de porter au-delà des quelques cercles d'économistes assez minoritaires dont il fait partie. Et l'on serait en effet pour le moins désireux de savoir ce qu'en pensent les économistes encore nombreux que la crise passée n’a nullement «atterrés» et pour lesquels l'injonction à une refondation de la science économique ne revêt aucunement le caractère impératif que lui assigne André Orléan. 
Le dossier qui suit offre un regard croisé et interdisciplinaire sur l'ouvrage d’André Orléan à travers trois contributions critiques émanant d'un sociologue, Philippe Steiner, d'un économiste, Richard Arena, et d'un philosophe, Marc Hunyadi. Il est l'occasion d'un éclairage nouveau et d'une discussion critique et argumentée à l'endroit de quelquesunes des thèses refondatrices de L'Empire de la valeur.

- Philippe Steiner entreprend de montrer que l'analyse des échanges marchands à laquelle se livre André Orléan n’est pas sans faire écho aux développements contemporains de la sociologie relationnelle et qu'elle pose, ce faisant, les jalons d'une véritable sociologie économique relationelle.

- Richard Arena entend, de son côté, mettre les thèses d’André Orléan à l'épreuve d'une analyse en terme de coût et de répartition des revenus. Il vise ainsi à intégrer la dimension productive et technologique du système de production capitaliste dans le projet de redéfinition de la valeur, par delà la seule composante marchande de l'échange.

- Marc Hunyadi, enfin, soulève une ambiguïté fondamentale dans l'analyse marxiste de la valeur-travail et vise à contester la thèse d’André Orléan selon laquelle Marx aurait fait à son tour du travail, dans le cours même de sa critique du fétichisme de la valeur, une substance. 